\title{
Pneumomediastinum secondary to invasive and non-invasive mechanical ventilation
}

\author{
Sara Mousa MD, Hawa Edriss MD
}

\begin{abstract}
Pneumomediastinum (PM) is defined as the presence of abnormal gas in the mediastinum. It is a known complication of invasive mechanical ventilation and has been reported with non-invasive ventilation. Recent studies have reported that the incidence of barotrauma is lowest in post-operative patients and is highest in mechanically ventilated patients with acute respiratory distress syndrome. The incidence has dropped with the low tidal volume ventilation technique. Chest $x$-rays can miss up to $25 \%$ of small PMs detected by computed tomography scans of the chest. Pneumomediastinum is managed with low tidal volume ventilation with plateau pressures $<30 \mathrm{~cm} \mathrm{H}_{2} \mathrm{O}$ and treatment of the underlying lung disease. Novel ways of ventilation, such as high frequency oscillatory ventilation and asynchronous independent lung ventilation, may improve ventilation in some patients.
\end{abstract}

Keywords: Pneumomediastinum, barotrauma, mechanical ventilation, air leak, ventilator induced lung injury

\section{INTRODUCTION}

Pneumomediastinum (PM) is defined as the presence of abnormal gas in the mediastinum. Air leakage from ruptured alveoli into the mediastinum can occur spontaneously, or following trauma, invasive procedures, and thoracic surgery, or during invasive mechanical ventilation (IMV). Non-invasive ventilation (NIV) has also been reported to cause PM. Rupture of the alveoli leads to leakage of air along bronchovascular bundles into the hilum and from there into the mediastinum. Along with PM, pneumothorax, subcutaneous emphysema, and sometimes pneumoperitoneum are known complications associated with mechanical ventilation (MV) and are collectively called barotrauma. A decrease in lung compliance, e.g., acute respiratory

Corresponding author: Sara Mousa

Contact Information: Sara.mousa@ttuhsc.edu DOI: 10.12746/swrccc.v7i27.524 distress syndrome (ARDS), age, and underlying lung diseases, such as interstitial lung disease (ILD), chronic obstructive pulmonary disease (COPD), cystic fibrosis, and certain lung infections like Pneumocystis jiroveci pneumonia (PJP), are risk factors for PM.

\section{INCIDENCE}

Laennec first described PM in 1819, and Hamman described spontaneous PM in 1939. ${ }^{1}$ Hoo et al reported the incidence of barotrauma was the lowest in post-operative patients $(0.5 \%)$ and the highest in mechanically ventilated ARDS patients (86\%). ${ }^{2}$ Patients with ARDS had a higher incidence of barotrauma than patients with other diagnoses (40-60\%) with a similar mortality range. ${ }^{2}$ Caceres et al reported higher mortality with PM due to barotrauma induced by MV than with PM due to other conditions not caused by MV. The incidence has decreased with low tidal volume mechanical ventilation. ${ }^{3} \mathrm{De}$ Lattore reported the incidence of barotrauma in mechanically ventilated patients was 4\% (22 out of 
553 patients). He also reported the incidence of $\mathrm{PM}$ and pneumothorax was $38 \%$ (6 out of 16 patients) in invasively ventilated patients with aspiration pneumonia. This higher incidence was attributed to necrosis in the bronchoalveolar structures injured by aspiration. ${ }^{4}$ Perez et al observed no difference in the incidence of air leak syndromes in two groups of preterm infants treated with surfactant managed with either continuous positive airway pressure (CPAP) or invasive ventilation. ${ }^{5}$ No data comparing PM caused by invasive vs non-invasive ventilation in adults were found for this review.

\section{Pathophysiology}

Mechanical ventilation can be complicated by PM, which is one of the manifestations of barotrauma which also includes pneumothorax, subcutaneous emphysema, and pneumoperitoneum. Barotrauma refers to increased stress and pressure in the alveoli and is one of the different presentations of ventilatorinduced lung injury (VILI). Other presentations of VILI include volutrauma, atelectrauma, and biotrauma. Volutrauma refers to alveolar over-distension and strain. Atelectrauma is the injury to the lung caused by sheer forces secondary to cyclic alveolar collapse and reopening, and biotrauma refers to extensive inflammatory response in the lungs secondary to mechanical ventilation. ${ }^{3,6}$ Regional lung tissue has non-homogenous susceptibility to ventilator forces. This local lung damage has been attributed to anisotropic alveolar inflation when the alveoli open during inspiration and collapse at the end of expiration. Some regions of the lung are more susceptible to volutrauma than others. Non-dependent parts of the lung tend to have higher risk of rupture with high inspiratory pressure of tidal volume, while dependent parts of the lung are at risk of large stress with opening during inspiration and then collapsing during expiration with insufficient PEEP. ${ }^{6}$ Pneumocystis jiroveci pneumonia can cause cysts and pneumatoceles that might rupture and lead to PM. Weng et al retrospectively reviewed the medical records of 82 patients who were admitted to the ICU with nonHIV related PJ pneumonia and concluded that PM complicating PJ pneumonia carries higher mortality (21\% compared to $5 \%$ in patients without PM). ${ }^{7}$ The pathophysiologic process that connects the steps of alveolar rupture, air leakage along the bronchovascular sheaths, and then escape into the mediastinum and at times subcutaneous tissues is known as the Macklin effect. This effect is usually detected in chest computed tomography (CT) scans.

The hemodynamic effects of PM were studied by Tulapati; they measured changes in heart rate $(H R)$, blood pressure (BP), mean arterial blood pressure (MAP), central venous pressure (CVP), and the size of mediastinal air column in lateral chest radiography (CXR) after inserting a mediastinal chest tube to drain the air in 25 pediatric patients. The mean change in the mediastinal air column was $-1.6 \mathrm{~cm}$. The change in HR was -4 beats/minutes, systolic $\mathrm{BP}+16 \mathrm{mmHg}$, diastolic $\mathrm{BP}+11 \mathrm{mmHg}, \mathrm{MAP}+12 \mathrm{mmHg}$, and CVP $-2 \mathrm{mmHg}$. In four patients, the cardiac index was measured after mediastinal tube insertion, and it increased $34 \%$. No complications recorded with this intervention. ${ }^{8}$ No similar data in adults were found for this review.

Repetitive stretch of the lung caused by MV causes VILI. Boudreault et al identified adaptive molecules that help protect the lung against stress at a cellular level. Those molecules are mettalothionein and intracellular zinc. Mettalothionein is an antioxidant and cytoprotective molecule that maintains cellular zinc homeostasis. Its expression is greatly affected by zinc levels which are mainly influenced by dietary intake. They applied stress to the lung in a form of high tidal volumes to mice lungs in vivo and stretch to cultured human lung cells. During these maneuvers, increased expression of mettalothionein was detected. Also in this study, zinc deficiency was induced in a group of mice using a zinc deficient diet. Zinc deficient mice more frequently developed ventilator-induced lung injury than control mice on a regular diet. They also found that zinc levels in patients who developed ARDS were lower than in other ICU patients without ARDS and in healthy patients. ${ }^{9}$

\section{Diagnosis}

Air leaks from ruptured alveoli into the mediastinum and can be detected radiologically before these leaks present with clinical symptoms. Air accumulates 


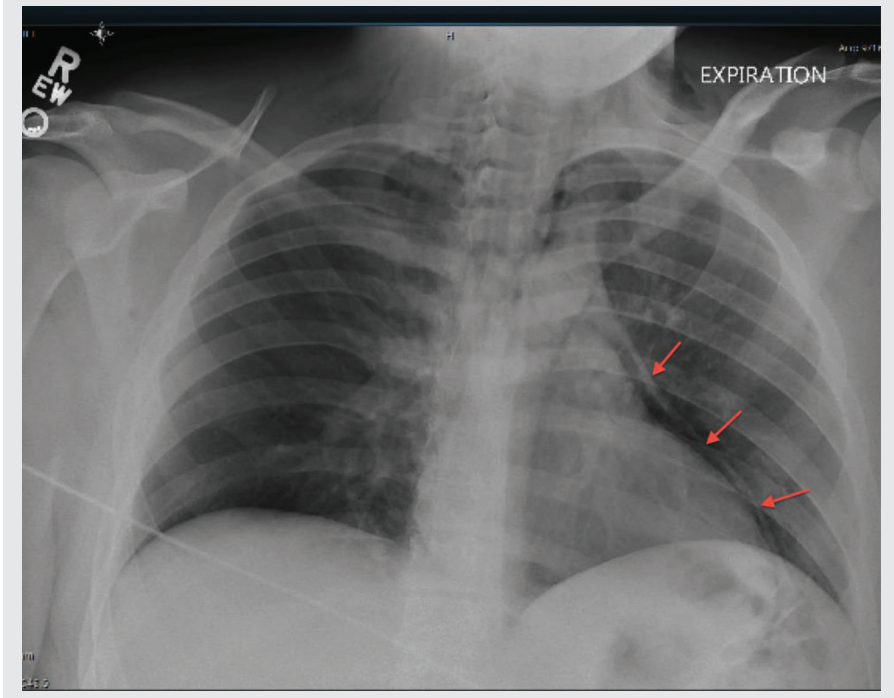

Figure 1. Patient presented with fever and tachycardia and imaging showed PM with air around the heart (arrows).

around the heart, in the retrosternal area, and around the trachea. Chest $x$-rays (CXR) and CT scans of the chest are the main methods used to diagnose PM (Figures 1-4). Okada concluded that CXR can miss up to $25 \%$ of small PM cases detected on CT scans. In his study CXRs were reviewed retrospectively

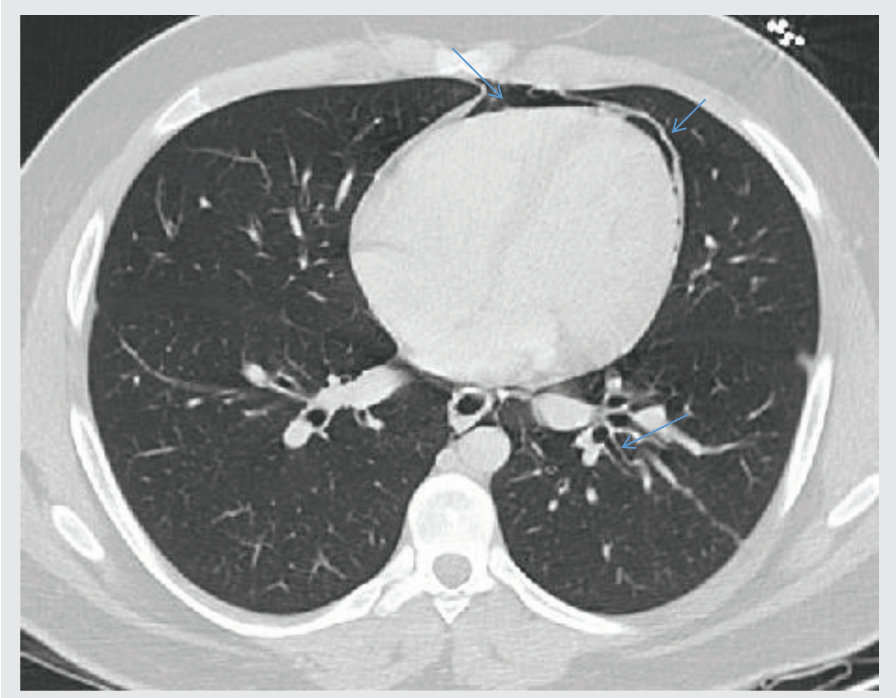

Figure 2. Air around the heart (arrows) (same pateint in Figure 1).

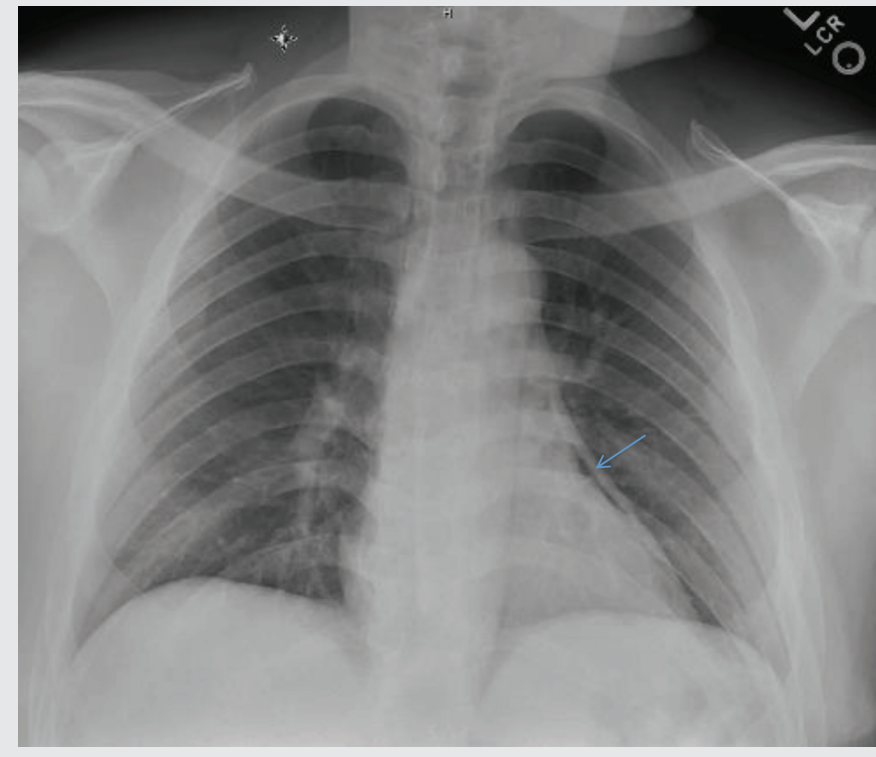

Figure 3. Air around the heart (arrow) in an asthmatic patient who presented 2 days following an acute asthma exacerbation.

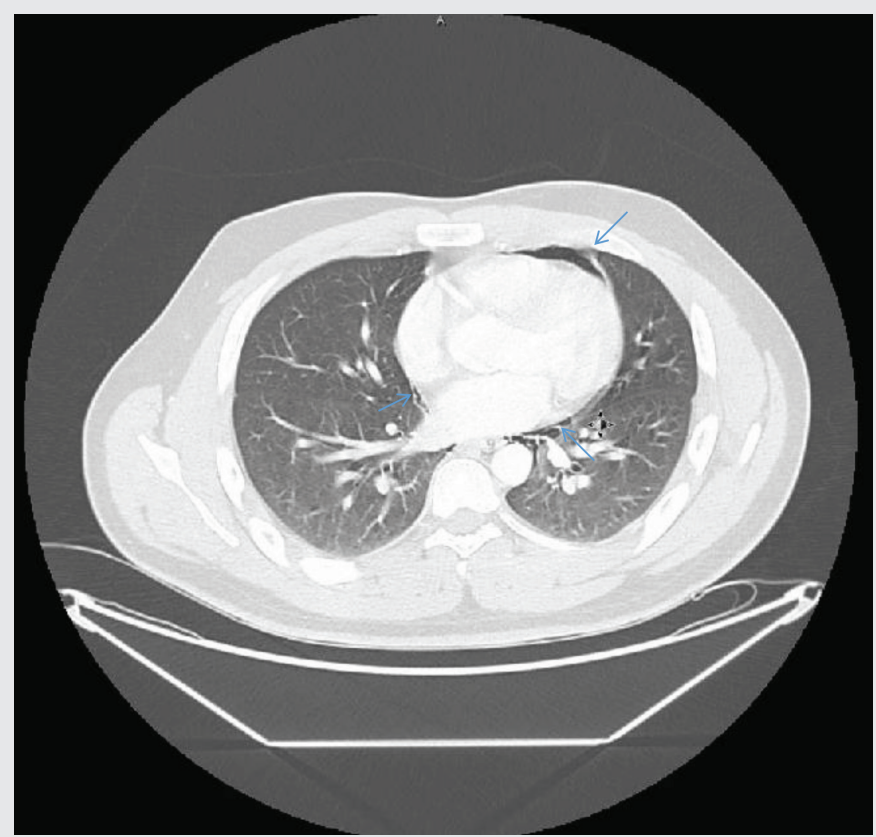

Figure 4. Air in the mediastinum surround the heart (arrows) (same patient in Figure 3). 
in 20 patients who presented with spontaneous PM. Five patients had negative CXR, but 64-slice chest CT scans showed the Macklin effect in all 20 patients. ${ }^{10}$ Wong et al suggested that adding lateral neck radiographs can help detect PM in patients with normal CXRs and found free air in neck soft tissue in 9 out of 10 patients with spontaneous PM and a normal CXR. ${ }^{11} \mathrm{Wu}$ et al suggested it was unnecessary to use fluoroscopic esophagograms to detect esophageal perforation since chest CTs with contrast have the same or higher sensitivity and negative predictive values as esophagograms. ${ }^{12}$ Ho et al compared the findings in multidetector CT scans in patients with spontaneous PM and secondary PM and noted that air is more likely detected in the anterior mediastinum in spontaneous than in secondary PM. He also noted that pleural effusions, acute pulmonary airspace opacities, and sub-diaphragmatic air are more likely in secondary PM than in spontaneous PM. ${ }^{13}$

\section{ClinICAL SERIES}

Umegaki et al retrospectively reviewed data on 149 ARDS patients who were mechanically ventilated and showed that a peak pressure of $>35 \mathrm{mmHg}$ increased the risk of barotrauma. Many patients who develop ARDS have comorbidities that are treated with corticosteroids. Umegaki suspected that there might be an association between corticosteroid use prior to admission and barotrauma. He retrospectively compared patients who developed barotrauma on mechanical ventilation who had ARDS and had been treated with corticosteroids before admission and patients who had not received these drugs. There was no significant difference in the incidence of barotrauma. ${ }^{14}$ Yang et al analyzed clinical data from patients with severe acute respiratory syndrome (SARS) and found that 7 out of 27 of patients with SARS on NIV developed PM in contrast to 1 of 189 of patients with SARS on no mechanical ventilation. ${ }^{15}$

Zhang et al analyzed the survival in 28 patients with connective tissue disease who developed PM. His analysis showed that $86 \%$ of patients who survived a month after the PM survived more than 1 year. He reported better prognosis in patients with high albumin levels, in patients with "slow" air leaks, and in patients who were started on immunosuppressive treatment with in one month of starting corticosteroid therapy. He also reported that the rate of air leak in patients with connective tissue disease complicated by PM has remarkable impact on mortality independent of other factors. A slow air leak rate was defined as the time from the development of PM to the onset of dyspnea defined by either the new onset respiratory failure needing ventilator support or by drop in $\mathrm{PaO}_{2}>30 \mathrm{mmHg}$ after $\mathrm{PM}$ greater than 3 days. ${ }^{16}$ Colombi et al retrospectively compared two groups of patients, namely ILD with PM and ILD without PM. Survival was analyzed, and PM was a strong predictor of mortality with a hazard ratio of $3.0(p=0.032) .{ }^{17}$

\section{Management AND OUTCOMES}

Management of PM caused by MV requires continued mechanical ventilation but with settings that are less injurious to the lung, if possible. Low tidal volumes of $4-8 \mathrm{ml} / \mathrm{kg}$ and plateau pressures less than $30 \mathrm{~cm}$ $\mathrm{H} 2 \mathrm{O}$, especially in patients with ARDS, are lung protective. Treating the underlying lung disease is also necessary. Some case reports have described the use of high frequency oscillatory ventilation and asynchronous independent lung ventilation. Others reported that use of extracorporeal membrane oxygenation (ECMO).

Some patients with spontaneous PM can be treated as outpatients. Ebina et al reported that 23 patients out of $34(67.6 \%)$ with spontaneous PM required admission. These patients were admitted for observation, treatment of an asthma exacerbation, respiratory failure after Influenza infection, or observation after childbirth; only one patient had recurrent PM. This result supports a strategy of treating some patients with spontaneous PM as outpatients unless there is doubt about the underlying cause or if the cause needs specific medical treatment or needs further workup. ${ }^{18}$

\section{ILLUSTRATIVE CASES-CLINICAL DISORDERS}

Any deterioration in a patient's condition while on NIV should raise suspicion of possible barotrauma, especially in patients with underlying lung diseases. Garcia et al reported a 12-year-old girl who developed 
PM after being admitted with status asthmaticus and put on NIV. ${ }^{19}$

Pneumomediastinum can progress rapidly in patients with underlying ILD despite aggressive medical treatment of underlying lung disease. Bakhshaee and co-workers reported a patient with dermatomyositis on immunosuppressive medications who presented with neck pain and shortness of breath and a CT scan of the chest showed ground glass infiltrates, PM, and pneumothorax. This patient died despite intubation and aggressive immunosuppression. ${ }^{20}$

Pneumomediastinum can cause fatal pericardial tamponade; this is attributed to peak inspiratory pressure increases if the patient is coughing while on NIV. Ruggeri reported fatal PM in a 78-year-old patient with an acute exacerbation of COPD and ILD, which was treated with NIV who developed pericardial tamponade as the cause of his death. ${ }^{21}$

Jonathan reported a case of intraoperative cardiac arrest secondary to tension PM in a patient with acute intracranial bleed secondary to moyamoya syndrome. This patient went into respiratory collapse, and most causes of circulatory collapse were ruled out with arterial blood gases, intraoperative trans-esophageal echocardiogram, and bronchoscopy. Postoperative CT chest ruled out pulmonary embolism. However, it did show PM as a rare cause of intraoperative circulatory collapse. ${ }^{22}$

Bronchiolitis obliterans, bronchiolitis obliterans with organizing pneumonia, and non-classifiable interstitial pneumonia are known lung complications in graft versus host disease (GVHD). Those complications can cause extra-alveolar air leaks called the thoracic air leak syndrome. Colin el al reported a case of tension PM in a patient with lung manifestations of GVHD consistent with non-classifiable interstitial pneumonia after allogeneic hematopoietic stem cell transplantation for non-Hodgkin lymphoma. ${ }^{23}$ He developed air leak syndrome and invasive pulmonary aspergilosis and died. Air leak syndrome usually has a poor prognosis in these patients.

\section{ILLUSTRATIVE CASES-TREATMENT}

McGinley et al reported the use of high frequency oscillatory ventilation in an 18-month-old child who survived staphylococcal tracheitis and necrotizing pneumonitis complicated by PM. ${ }^{24}$

Mehdiratta et al reported a case of PM and tracheobronchial disruption secondary to blunt trauma that was treated with asynchronous independent lung ventilation due to unilateral lung contusion. Initially double lumen endotracheal tube placement was attempted but could not be inserted due to airway edema. Later the patient became hypoxemic, and emergent cricothyroidotomy was initiated; however, single lumen endotracheal (ETT) intubation was placed before completion of the cricothyroidotomy. Due to the location of the laceration, the single tube could not isolate the defect. Then decision to proceed with traditional tracheostomy with selective intubation of the right mainstem with a $5.0 \mathrm{~mm}$ cuffed ETT, and the left mainstem with a $6.0 \mathrm{~mm}$ ETT. The contused lung was ventilated using airway pressure release ventilation, and the other lung was ventilated using standard ventilation. After few days on asynchronous ventilation, the contused lung improved, and standard ventilation was used with both lungs. ${ }^{25}$

Ali and co-workers reported a patient with $\mathrm{PJ}$ pneumonia complicated by PM while on NIV who then needed invasive MV. Despite the MV patient continued to have severe hypoxemia and was then managed with veno-venous extra-corporeal membrane oxygenation ( $\mathrm{V}-\mathrm{V} \mathrm{ECMO}$ ) to help maintain adequate oxygenation while his infection was being treated. ${ }^{26}$

Tension PM can present with complications, such as cardiac arrest, that require decompression to relieve the pressures around the heart. The cause of PM usually determines the location of the air collection in the mediastinum. Pneumomediastinum due to alveolar rupture usually collects in the anterior mediastinum, while PM due to esophageal rupture usually accumulates in the posterior mediastinum. Paluszkiewicz et al reported a case of tension PM as a delayed complication of Boerhaave syndrome that was complicated by cardiac arrest. Decompression through cervical and subxiphoid mediastinostomy was unsuccessful. Decompression of the lower periesophageal space through the esophageal hiatus was successful, and sinus rhythm returned immediately afterwards. This case suggests that decompression 
of the posterior mediastinum in cases with failed classical anterior mediastinal decompression can reduce the tension. ${ }^{27}$

\section{Conclusions}

1. The incidence of PM in mechanically ventilated patients is higher in ARDS and has decreased with the low tidal volume ventilation.

2. Chest $x$-rays can miss up to $25 \%$ of small $P M s$ detected by CT scans of the chest.

3. Pneumomediastinum is managed with low tidal volume ventilation with plateau pressures less than $30 \mathrm{~cm} \mathrm{H}_{2} \mathrm{O}$ and treatment of underlying lung disease. Novel ways of ventilation, such as high frequency oscillatory ventilation and asynchronous independent lung ventilation, may improve ventilation in some patients.

4. Spontaneous PM can often be managed as an outpatient unless the underlying cause needs medical treatment or further workup.

Article citation: Mousa S, Edriss H. Pneumomediastinum secondary to invasive and non-invasive mechanical ventilation. The Southwest Respiratory and Critical Care Chronicles 2019;7(27):36-42

From: The Department of Internal Medicine at Texas Tech University Health Sciences Center in Lubbock, Texas

Submitted: $5 / 1 / 2018$

Accepted: $1 / 4 / 2019$

Reviewer: Victor Test MD

Conflicts of interest: none

This work is licensed under a Creative Commons

Attribution-ShareAlike 4.0 International License.

\section{REFERENCES}

1. Gasser CR, Pellaton R, Rochat CP. Pediatric spontaneous pneumomediastinum: narrative literature review. Pediatric Emergency Care 33(5):370-374.

2. Hoo GW. Barotrauma and mechanical ventilation. emedicine. medscape.com/article/296625-overview. Updated: Apr 18, 2018.
3. Caceres M, Braud R L, Maekawa R, et al. Secondary pneumomediastinum: A retrospective comparative analysis. Lung 2009 Sep-Oct;187(5):341-6.

4. De Latorre FJ, Klamburg J J, Leon C, et al. Incidence of pneumothorax and pneumomediastinum in patients with aspiration pneumonia requiring ventilatory support. Chest 1977 Aug;72(2):141-4.

5. Pérez LA, González DM, Álvarez KM, et al. Nasal CPAP versus mechanical ventilation in 28 to 32 -week preterm infants with early surfactant administration. Biomedica 2014 Oct-Dec;34(4):612-23.

6. Loza RC, Rodríguez GV, Fernández NM. Ventilator-induced lung injury (VILI) in acute respiratory distress syndrome: volutrauma and molecular effects. Open Respir Med J 2015 Jun 26;9:112-9.

7. Weng L, Huang X, Chen L, et al. Prognostic factors for severe Pneumocystis jiroveci pneumonia of non-HIV patients in intensive care unit: a bicentric retrospective study. $B M C$ Infect Dis. 2016;16(1):528.

8. Turlapati KM, Spear RM, Paterson BM. Mediastinal tube placement in children with pneumomediastinum: hemodynamic changes and description of technique. Crit Care Med 1996 Jul;24(7):1257-60.

9. Boudreault F, Pinilla-Vera M, Englert JA, et al. Zinc deficiency primes the lung for ventilator-induced injury. JCI Insight 2017 Jun 2;2(11). pii: 86507. doi: 10.1172/jci.insight.86507.

10. Okada M, Adachi H, Shibuya Y, et al. Diagnosis and treatment of patients with spontaneous pneumomediastinum. Respir Investig 2014 Jan. 52(1):36-40.

11. Wong KS, Wu HM, Lai SH, et al. Spontaneous pneumomediastinum: analysis of 87 pediatric patients. Pediatr Emerg Care 2013 Sep. 29(9):988-91.

12. $\mathrm{Wu} \mathrm{CH}$, Chen $\mathrm{CM}$, Chen $\mathrm{CC}$, et al. Esophagography after pneumomediastinum without $\mathrm{CT}$ findings of esophageal perforation: is it necessary?. AJR Am J Roentgenol 2013 Nov. 201(5):977-84.

13. Ho AS, Ahmed A, Huang JS, et al. Multidetector computed tomography of spontaneous versus secondary pneumomediastinum in 89 patients: can multidetector computed tomography be used to reliably distinguish between the 2 entities? J Thorac Imaging 2012 Mar. 27(2):85-92.

14. Umegaki T, Kenichiro S, Akihisa N, et al. Impact of steroid medication before hospital admission on barotrauma in mechanically ventilated patients with acute respiratory distress syndrome in intensive care units. J Anesth 2014 Oct;28(5):681-6.

15. Yang $L, L i F, L i D$, etal.Clinical analysis of complicationsafter non- invasive positive pressure ventilation and an inquiry into the respiratory treatment strategy in patients with SARS. Zhongguo Wei Zhong Bing Ji Jiu Yi Xue 2004 May;16(5): $281-3$. 
16. Zhang L, Shen M, Zhang F, et al. Survival analysis and risk factors for mortality in connective tissue diseaseassociated pneumomediastinum. Rheumatol Int 2014 Dec; 34(12):1657-63.

17. Colombi D, Ehlers-Tenenbaum S, Palmowski K, et al. Spontaneous pneumomediastinum as a potential predictor of mortality in patients with idiopathic pulmonary fibrosis. Respiration 2016;92(1):25-33.

18. Ebina M, Inoue A, Takaba A. Management of spontaneous pneumomediastinum: Are hospitalization and prophylactic antibiotics needed? Am J Emerg Med 2017 Aug;35(8): 1150-1153.

19. García LG, Corsino Rey, Medina A, et al. Severe subcutaneous emphysema and pneumomediastinum secondary to noninvasive ventilation support in status asthmaticus. Indian J Crit Care Med 2016 Apr;20(4):242-4.

20. Bakhshaee M, Jokar MH, Mirfeizi Z, et al. Subcutaneous emphysema, pneumomediastinum and pneumothorax in a patient with dermatomyositis. Iran J Otorhinolaryngol 2017 Mar; 29(91):113-116.

21. Ruggeri P, Girbino G. Fatal pneumomediastinum associated with use of noninvasive mechanical ventilation. Respirol Case Rep 2014 Dec;2(4):126-8.
22. Weaver JB, Kumar AB. Tension pneumomediastinum: rare cause of acute intraoperative circulatory collapse in the setting of unremarkable TEE finding. J Clin Anesth 2017 Feb; 37:136-138.

23. Colin GC, Ghaye B, Coche E Tension pneumomediastinum secondary to thoracic air-leak syndrome in chronic graft versus host disease. Diagn Interv Imaging 2014 Mar;95(3):317-9.

24. McGinley J, Corcoran T, Canny G, et al. A case of pneumomediastinum in paediatric ARDS: to oscillate or not? Paediatr Anaesth 2001 May;11(3):366-9.

25. Mehdiratta N, Archer M, Stewart M, et al. Novel airway and ventilator management of tracheobronchial disruption after blunt trauma. Ann Thorac Surg 2017 Nov;104(5):e359-e361.

26. Ali HS, Hassan IF, George S. Extra corporeal membrane oxygenation to facilitate lung protective ventilation and prevent ventilator-induced lung injury in severe Pneumocystis pneumonia with pneumomediastinum: a case report and short literature review. BMC Pulm Med 2016 Apr 14;16(1):52. doi: 10.1186/s12890-016-0214-4.

27. Paluszkiewicz P, Bartosinski J, Rajewska-Durda K, et al. Cardiac arrest caused by tension pneumomediastinum in a Boerhaave syndrome patient. Ann Thorac Surg 2009 Apr;87(4): 1257-8. 